\title{
Long-distance dispersal and recombination in environmental populations of Cryptococcus neoformans var. grubii from India
}

Correspondence

Jianping Xu

.jpxu@mcmaster.ca

Received 4 December 2007

Revised 19 February 2008

Accepted 19 February 2008

\author{
Sanjay S. Hiremath, ${ }^{1}$ Anuradha Chowdhary, ${ }^{2}$ Tusharantak Kowshik, ${ }^{2}$ \\ Harbans S. Randhawa, ${ }^{2}$ Sheng Sun ${ }^{1}$ and Jianping $\mathrm{Xu}^{1}$
${ }^{1}$ Center for Environmental Genomics, Department of Biology, McMaster University, Hamilton, ON L8S 4K1, Canada \\ ${ }^{2}$ Department of Medical Mycology, Vallabhbhai Patel Chest Institute, University of Delhi, \\ 110007 Delhi, India
}

\begin{abstract}
The basidiomycete yeast Cryptococcus neoformans is a cause of significant morbidity and mortality in immunocompromised hosts throughout the world. The sporadic nature of the infection and the limited empirical evidence for direct human-to-human transmission have led to the belief that infections in humans are predominantly caused by the inhalation of basidiospores from environmental sources. Therefore, analysing the structure of environmental populations of $C$. neoformans can significantly increase our understanding of its ecology, evolution and epidemiology. Decaying wood is a rich source of organic and inorganic compounds and is known to be a suitable ecological niche for many micro-organisms, including C. neoformans. However, relatively little is known about the population structure of $C$. neoformans sampled from decaying wood. In this study, we analysed samples of $C$. neoformans var. grubii colonizing decaying wood in tree hollows of nine tree species in five geographical locations (Delhi, Bulandshahar, Hathras, Amritsar and Amrouli) in north-western India. Multilocus sequence typing was conducted using five gene fragments for each of 78 isolates. All isolates belonged to mating type $\alpha$. Population-genetic analyses identified no evidence for significant differentiation among populations belonging to either different geographical areas or different host tree species. Interestingly, despite the lack of mating type a strains in our survey, we found unambiguous evidence for recombination in our population analyses. Our results are consistent with the hypothesis of long-distance dispersal and recombination in environmental populations of this species in India.
\end{abstract}

\section{INTRODUCTION}

Cryptococcus neoformans var. grubii is the most common causative agent of cryptococcosis and can lead to significant morbidity and mortality in hosts with a defective T-cell-mediated immunity (Casadevall \& Perfect, 1998). It has a global distribution and has been isolated from both human and environmental sources such as avian guano, soil, fruits, vegetables and decaying hollows of more than ten species of trees in different parts of the

Abbreviation: T-PTP, topology-dependent permutation tail probability.

The GenBank accession numbers for DNA sequences obtained here are: CAP1, EU312204-EU312281; FTR1, EU312282-EU312359; ITS, EU312360-EU312437; LAC, EU312438-EU312515; mtLrRNA, EU312516-EU312593.

A supplementary figure showing maximum-parsimony trees for the $L A C$, CAP1 and FRT1 markers is available with the online version of this paper. world (Randhawa et al., 2003; Reimão et al., 2007). Current surveys suggest that the prevalence of cryptococcosis caused by strains of $C$. neoformans var. grubii varies among geographical regions, probably a reflection of their variable frequencies in different environments (Bennett et al., 1977; Casadevall \& Perfect, 1998; Tintelnot et al., 2004). C. neoformans var. grubii represents strains of serotype A, one of the five serotypes (A, B, C, D and AD) defined based on their cell-surface antigenic properties within the pathogenic cryptococcal species complex.

Like those of other micro-organisms in nature, environmental conditions for $C$. neoformans may change and fluctuate significantly, both spatially and temporally, in nutrient levels, temperature, water availability, etc. Under conditions of low nutrient availability, low moisture and a temperature in the range of $15-33{ }^{\circ} \mathrm{C}$, mating and sexual reproduction between strains of opposite mating types may occur, resulting in the production of basidiospores. These 
sexual spores are smaller than vegetative cells and can disperse easily by wind or other means to other environmental niches, including human hosts.

The mating system of this heterothallic basidiomycete is controlled by one locus with two alternative alleles: MATa and MATa (Kwon-Chung, 1975, 1976). Previous studies have shown that the majority of clinical and environmental isolates belong to the MAT $\alpha$ mating type (Kwon-Chung \& Bennett, 1978; Yan et al., 2002). Interestingly, a laboratory $M A T \alpha$ strain that belonged to serotype D (one of three serotypes, $\mathrm{A}, \mathrm{D}$ and $\mathrm{AD}$, in C. neoformans) has been shown to be more virulent than its congenic MATa strain in a murine model of cryptococcosis (Kwon-Chung et al., 1992). Although the details and the relative frequencies of mating in natural environments are little known, in the laboratory, both opposite-sex mating $(M A T a-M A T \alpha)$ and same-sex mating (MAT $\alpha-M A T \alpha)$ have been demonstrated (Kwon-Chung, 1976; Keller et al., 2003; Nielsen et al., 2003; Lin et al., 2005). A recent study indicated that the MATa locus was among several genomic regions that contribute quantitatively to same-sex mating and haploid fruiting in C. neoformans (Lin et al., 2006). Evidence for same-sex mating $(M A T \alpha-M A T \alpha)$ in nature was recently reported between strains of serotypes A and D (Lin et al., 2007). Same-sex mating has also been hypothesized to be responsible for generating the major genotype of a recent outbreak of cryptococcosis on Vancouver Island, British Columbia, Canada (Fraser et al., 2005).

Sexual reproduction, between strains either of opposite sexes or the same sex, can potentially increase genetic variation in natural populations of organisms. The diversity of genotypes resulting from sexual reproduction can increase the efficiency of natural selection and may enhance the chances of successfully colonizing novel environments (Weismann, 1904; Goddard et al., 2005). However, unlike those in the majority of plants and animals, where sexual reproduction can be easily detected, the detection of mating and sexual reproduction in microorganisms requires the analysis of genotypic data from multiple strains in populations. Associations among alleles from within the same or different loci are used as indicators for sexual or asexual reproduction, typically through the analyses of multilocus linkage disequilibrium (Xu et al., 1999; Xu, 2005b). In these tests, random associations (i.e. linkage equilibrium) among alleles at the population level are consistent with sexual reproduction while significant associations (linkage disequilibrium) are indicative of clonal reproduction. Other signatures of clonal reproduction include over- or under-representations of certain genotypes and genealogical congruence among genes from unlinked loci (e.g. Xu, 2005b).

Previous studies have employed a variety of molecular markers to demonstrate the modes of microbial reproduction in nature and to determine how evolutionary as well as ecological factors can influence the modes of reproduction and the structures of microbial populations (Maynard-Smith et al., 1993, Avise, 1994; Xu, 2005b), including those of $C$. neoformans. For example, an MLST study of the population structure of C. neoformans var. grubii involving 102 representative strains from different parts of the world showed that the global population contained three genetically distinct subgroups (Litvintseva et al., 2006). Ten of the 102 strains were MATa and they were all from Botswana. The remaining 92 were MAT $\alpha$ and were from different parts of the world, including Botswana. As expected, the Botswana population that contained strains of both mating types showed evidence of recombination and sexual reproduction (Litvintseva et al., 2003, 2006). In contrast, the remaining geographical populations that contained only MAT $\alpha$ strains were largely clonal, with no clear evidence of recombination. Similar analyses of natural populations of a closely related species, Cryptococcus gattii, that contained exclusively or predominantly MAT $\alpha$ strains identified at least three distinct lineages in this species but no conclusive evidence for recombination (Kidd et al., 2005). However, in another study of C. gattii populations, evidence for both clonality and recombination was obtained, with clonality found for samples belonging to one molecular type, VGI, and evidence of recombination found for samples of a different molecular type, VGII (Campbell et al., 2005).

In this study, we analysed 78 strains of $C$. neoformans var. grubii isolated from nine tree species distributed in five geographical areas in north-western India. The mating types of these strains were determined based on direct PCR using mating-type-specific primers (Yan et al., 2002) and all strains were found to have the MAT $\alpha$ mating type. A multiple-gene genealogical approach was used to analyse the population samples. DNA sequences were obtained for each strain from each of the five genetic loci distributed in different parts of the genome. Our population-genetic analyses identified no evidence of population subdivision based on either the host tree species or their geographical origins. Interestingly, while clonality and clonal dispersal among geographical regions were clearly evident in our analysed populations, there was also unambiguous evidence for recombination in this MATa population of $C$. neoformans var. grubii.

\section{METHODS}

Samples of $\boldsymbol{C}$. neoformans. The decaying wood inside the trunk hollows of nine species of trees was sampled for Cryptococcus neoformans (serotypes A and D) between 2003 and 2006. Strains were collected by taking swabs from the decayed wood and inoculating the swabs on modified niger seed agar (Randhawa et al., 2005). The colonies were screened for morphological (microscopy) and physiological characteristics consistent with C. neoformans (VITEK 2 system, bioMérieux). Isolates were then serotyped using the Crypto-check slide agglutination test (Iatron). Details of the 78 strains obtained for this study are presented in Table 1 . The strains analysed here are available upon request from Drs $\mathrm{H}$. Randhawa and J. Xu.

DNA extraction and PCR amplification. A previously described technique (Xu et al., 2000) was used to extract high-molecular-mass genomic DNA from all 78 strains. Fragments of four nuclear DNA 
genes and one mitochondrial gene were chosen for PCR amplification and analyses. To avoid the potential bias in linkage disequilibrium due to physical linkage between markers, the five marker loci used in this study were located on separate chromosomes, as determined based on the completed JEC21 genome (Loftus et al., 2005). The nuclear DNA markers used for amplification were: (1) CAP1 (on chromosome 4) located within the mating locus and encoding a capsule-synthesis-associated protein; (2) FTR1 (on chromosome 3), which encodes a high-affinity iron permease; (3) LAC (on chromosome 7), which encodes the laccase/diphenol oxidase; (4) the internal transcribed spacer (ITS) region, which comprises ITS1, 5.8S rRNA and ITS2 (on chromosome 2); and (5) the mitochondrial large-subunit RNA ( $m t L r R N A)$ (Xu et al., 2000). The JEC21 genome assembly from the Stanford Genome Technology Center (http:// www.tigr.org/tdb/e2 $\mathrm{kl} / \mathrm{cnal} /$ ) was used to identify the loci and design primers when required. Fragments of $731 \mathrm{bp}$ (CAP1), $788 \mathrm{bp}$ (FTR1), $486 \mathrm{bp}$ (LAC), $468 \mathrm{bp}$ (ITS) and $439 \mathrm{bp}$ ( $m$ tLrRNA) were obtained by using the following primer pairs $\left(5^{\prime} \rightarrow 3^{\prime}\right)$ : CAP1, CGTTCGCGATAGAGAGAGGA (forward) and CCTTACCTTCACAGTCGCCC (reverse); FTR1, GTTCTCGGTCACCATCTTC (forward) and TCTCAGGCTCGCCATCTTC (reverse); LAC, GGCGATACTATTATCGTA (forward) and TTCTGGAGTGGCTAGAGC (reverse); ITS, TCCGTAGGTGAACCTGCGG (forward) and TCCTCCGCTTATTGATATGC (reverse); mtLrRNA, CGCTAAATACGATCCTGCATG (forward) and TTATCCCTAGCGTAACTTTTATC (reverse). Each PCR reaction comprised $2.1 \mu \mathrm{l}(\sim 1 \mathrm{ng})$ template DNA, $8 \mu \mathrm{l}$ GoTaq (Taq DNA polymerase $+\mathrm{MgCl}_{2}$ at a final concentration of $1.5 \mathrm{mM}$, supplied by Promega,), and $0.2 \mu \mathrm{M}$ of each primer to make up a total volume of $16 \mu \mathrm{l}$. The thermocycling profile included an initial denaturing temperature of $95{ }^{\circ} \mathrm{C}$ for 4 min followed by 40 cycles of $95{ }^{\circ} \mathrm{C}$ for $1 \mathrm{~min}$, annealing temperature for $1 \mathrm{~min}$, and $72{ }^{\circ} \mathrm{C}$ for $1 \mathrm{~min}$. The final extension step was $72{ }^{\circ} \mathrm{C}$ for $7 \mathrm{~min}$. Annealing temperatures for amplifying the five loci were: $59.2{ }^{\circ} \mathrm{C}$ for $C A P 1$, $58.6{ }^{\circ} \mathrm{C}$ for FTR1, $50{ }^{\circ} \mathrm{C}$ for $L A C, 50{ }^{\circ} \mathrm{C}$ for $I T S$, and $46{ }^{\circ} \mathrm{C}$ for $m t L r R N A$. The resulting PCR products were purified using the MicroCLEAN kit (Microzone) and sequenced at the MoBix Laboratory, McMaster University, using an ABI3100 automated DNA sequencer.

Mating type determination using molecular methods. The mating types of isolates were determined by PCR amplification using primer pairs designed from the sequences of the mating-type-specific STE12 and STE20 genes. These primers were serotype- and/or mating-type-specific. The specific primer sequences, amplification conditions, gel electrophoresis and data screening followed those described by Yan et al. (2002).

Phylogenetic analysis. Sequences from the 78 strains for each gene locus were aligned using CLUSTAL_X version 2 (Thompson et al., 1997). The alignments were then imported into SeaView (Galtier et al., 1996) and edited manually. Orthologues for the five genes in two model strains were retrieved for comparison from the completed genomes of serotype A (H99 strain, http://www.broad.mit.edu/ annotation/genome/Cryptococcus_neoformans/Home.html) and serotype D (JEC21 strain, http://www.tigr.org/). Phylogenetic analysis was performed using PAUP*4.0b10 (Swofford, 1996). Maximumparsimony trees were constructed for all the individual fragments by conducting heuristic searches based on 500 random sequence additions. Bootstrap analyses to assess the support for the clades were also conducted using 1000 replicate samples of phylogenetically informative characters. The serotype D strain JEC21 was used as an out-group for all the analysis. For the $L A C$ gene fragment, where extensive sequence information is available and is used to delineate the molecular subtypes (VNI, VNII and VNB) within C. neoformans var. grubii, strains representative of each of the three types from Litvintseva et al. (2003) were analysed together with our data.
Clonality and recombination. Three complementary tests were conducted to examine evidence for clonality and recombination in the Indian population of $C$. neoformans var. grubii. In the first test, we examined evidence for genealogical incongruence among the five genes. This test was conducted using the partition homogeneity test (PHT) or incongruence length difference (ILD) test (Farris et al., 1994) implemented in PAUP ${ }^{\star} 4.0 \mathrm{~b} 10$ (Swofford, 1996). The assumption made in this test is that the whole genome evolves as one single unit in strictly clonally reproducing organisms and as a result, genealogies of different gene fragments should be congruent in such organisms. In contrast, in the case of a recombining population structure, there would be incongruence among the different gene genealogies because different genes could have different histories. For this test, we used 100 replicates, each with 10 random sequence addition replicates using the NNI branch-swapping algorithm and saving a maximum of 1000 trees per replicate. This test was conducted for both the entire dataset and also the two largest individual populations, from Delhi and Amritsar, respectively. It has been shown that relying on this test alone for inferences of recombination could produce false-positive results when the consistency indices for individual gene trees are not high (Barker \& Lutzoni, 2002). To avoid this potential problem, we also conducted the following two population genetic tests for evidence of recombination.

In the second test, we examined allelic associations among alleles from different loci using two common statistics in population genetics: the index of association $\left(I_{\mathrm{A}}\right)$ (Maynard-Smith et al., 1993) and RbarD (Agapow \& Burt, 2001) tests. $I_{\mathrm{A}}$ and RbarD were estimated using Multilocus, version 1.0b (Agapow \& Burt, 2001). The $I_{\mathrm{A}}$ analyses the variance of the distances between all possible pairs of multilocus genotypes. The null hypothesis is that there is random association of alleles. Statistical significance was derived by comparing the observed dataset to 10000 artificially recombined datasets. During the process of generating the artificially recombined datasets, we permitted random shuffling of alleles at each locus within a population but kept the proportions of alleles at each locus constant. If there were linkage disequilibrium due to a lack of recombination, the observed $I_{\mathrm{A}}$ should be significantly higher than the randomized recombining dataset. RbarD is a modified measure of $I_{\mathrm{A}}$, but adjusted for the number of loci. The adjustment helps facilitate better comparison between populations and studies.

In the third test, we calculated the phylogenetic incompatibility by looking for the proportion of loci with all possible recombinant genotypes. This test looks for the evidence of recombination in a population by essentially looking for incongruence in different regions of the genome. The statistical significance of this test was inferred by comparing the number of incompatible pairs of loci in the observed dataset to those from a randomly shuffled dataset, using the program Multilocus, version 1.0b (Agapow \& Burt, 2001).

Population structure. We assessed the genetic structure of the Indian samples of $C$. neoformans using three different analyses. In the first, we conducted the T-PTP (topology-dependent permutation tail probability) test (Faith, 1991) to determine whether host tree species or the geographical locations of strains had a significant influence on the topologies of phylogeny. For this test, reshuffled datasets were generated using Multilocus version 1.0b (Agapow \& Burt, 2001) and the tree lengths were estimated using PAUP* version $4.0 \mathrm{~b} 10$ (Swofford, 1996). If there was no significant structure identified with respect to either host tree species or geographical origins, the optimal tree score for the observed data without any constraint would be significantly shorter than for trees with constrained topologies. In the second test, population differentiation analysis was done by calculating $\theta$ (Weir, 1996), using Multilocus version 1.0b (Agapow \& Burt, 2001). The null hypothesis of this test was no genetic differentiation between populations as defined by either host tree species or geographical 
Table 1. C. neoformans var. grubii strains used in this study

\begin{tabular}{|c|c|c|c|c|c|c|c|}
\hline \multirow{2}{*}{$\begin{array}{l}\text { Strain ID } \\
\text { INN3_279DDD_SC_DL_India } \dagger\end{array}$} & \multirow{2}{*}{$\begin{array}{l}\text { Source tree/geographical origin } \\
\text { Syzygium cumini/Delhi }\end{array}$} & \multirow{2}{*}{$\frac{\text { Year isolated }}{2003}$} & \multicolumn{5}{|c|}{ Multilocus genotype ${ }^{\star}$} \\
\hline & & & 1 & 1 & 1 & 1 & 2 \\
\hline INN4_280U-DDD_SC_DL_India & Syzygium cumini/Delhi & 2003 & 1 & 1 & 2 & 1 & 1 \\
\hline INN5_S284B_SC_DL_India & Syzygium cumini/Delhi & 2003 & 1 & 2 & 2 & 1 & 1 \\
\hline INN6_320-1DDD'_SC_DL_India $\dagger$ & Syzygium cumini/Delhi & 2004 & 1 & 3 & 1 & 1 & 1 \\
\hline INN8_320-2A_ii_PL_DL_India & Polyalthia longifolia/Delhi & 2004 & 1 & 4 & 2 & 1 & 1 \\
\hline INN9_320-3DDD'_PL_DL_India & Polyalthia longifolia/Delhi & 2004 & 1 & 1 & 2 & 1 & 1 \\
\hline INN11_320-4-DDD'_3_PL_DL_India $\dagger$ & Polyalthia longifolia/Delhi & 2004 & 1 & 3 & 2 & 1 & 1 \\
\hline INN12_322AA_1_PL_DL_India & Polyalthia longifolia/Delhi & 2004 & 1 & 3 & 2 & 1 & 1 \\
\hline INN13_322AA_2_PL_DL_India & Polyalthia longifolia/Delhi & 2004 & 1 & 3 & 2 & 1 & 1 \\
\hline INN14_323A-AA_PL_DL_India & Polyalthia longifolia/Delhi & 2004 & 1 & 1 & 2 & 1 & 1 \\
\hline INN15_323A-DDD_PL_DL_India & Polyalthia longifolia/Delhi & 2004 & 1 & 1 & 2 & 1 & 1 \\
\hline INN16_323B-AA_2_PL_DL_India & Polyalthia longifolia/Delhi & 2004 & 1 & 3 & 1 & 1 & 1 \\
\hline INN17_323B-DDD'_1_PL_DL_India & Polyalthia longifolia/Delhi & 2004 & 1 & 3 & 2 & 1 & 1 \\
\hline INN18_323B-DDD'_2_PL_DL_India & Polyalthia longifolia/Delhi & 2004 & 1 & 3 & 2 & 1 & 1 \\
\hline INN19_325A_PL_DL_India $\dagger$ & Polyalthia longifolia/Delhi & 2004 & 2 & 5 & 2 & 1 & 1 \\
\hline INN20_326B-DDD_1_PL_DL_India $\dagger$ & Polyalthia longifolia/Delhi & 2004 & 1 & 1 & 2 & 1 & 1 \\
\hline INN26_373DDD'_PL_DL_India & Polyalthia longifolia/Delhi & 2004 & 1 & 1 & 2 & 1 & 1 \\
\hline INN27_374A/2-10_PL_DL_India & Polyalthia longifolia/Delhi & 2004 & 1 & 1 & 2 & 1 & 1 \\
\hline INN28_374A/2-100_PL_DL_India & Polyalthia longifolia/Delhi & 2004 & 1 & 3 & 2 & 1 & 1 \\
\hline INN29_540S-BB_1_PL_DL_India & Polyalthia longifolia/Delhi & 2005 & 1 & 1 & 2 & 1 & 1 \\
\hline INN30_540S-BB_2_PL_DL_India & Polyalthia longifolia/Delhi & 2005 & 1 & 5 & 2 & 1 & 1 \\
\hline INN33_572S_ME_DL_India & Mimusops elengi/Delhi & 2005 & 1 & 1 & 2 & 1 & 1 \\
\hline INN34_572S-C_ME_DL_India & Mimusops elengi/Delhi & 2005 & 1 & 1 & 2 & 1 & 1 \\
\hline INN35_580A-d_ME_DL_India & Mimusops elengi/Delhi & 2005 & 2 & 3 & 2 & 1 & 1 \\
\hline INN36_580A-S_ME_DL_India & Mimusops elengi/Delhi & 2005 & 2 & 3 & 2 & 1 & 1 \\
\hline INN37_626S-C_AI_DL_India $\dagger$ & Azadirachta indica/Delhi & 2005 & 1 & 6 & 2 & 1 & 1 \\
\hline INN38_627d-BB_AI_DL_India $\dagger$ & Azadirachta indica/Delhi & 2005 & 2 & 1 & 2 & 1 & 1 \\
\hline INN39_628d-C_AI_DL_India $†$ & Azadirachta indica/Delhi & 2005 & 1 & 1 & 2 & 1 & 1 \\
\hline INN41_618A-d-C_MH_DL_India & Manilkara hexandra/Delhi & 2005 & 1 & 1 & 2 & 1 & 1 \\
\hline INN43_618A-d-BB_MH_DL_India & Manilkara hexandra/Delhi & 2005 & 1 & 1 & 2 & 1 & 1 \\
\hline INN44_618B-d-BB_MH_DL_India & Manilkara hexandra/Delhi & 2005 & 2 & 1 & 2 & 1 & 1 \\
\hline INN46_611d-C_1_AN_DL_India & Acacia nilotica/Delhi & 2005 & 1 & 1 & 2 & 1 & 1 \\
\hline INN48_692d-C_1_CF_DL_India & Cassia fistula/Delhi & 2005 & 1 & 3 & 1 & 1 & 1 \\
\hline INN49_692d-C_2_CF_DL_India $\dagger$ & Cassia fistula/Delhi & 2005 & 1 & 3 & 2 & 1 & 1 \\
\hline INN50_747S_2_CF_DL_India & Cassia fistula/Delhi & 2006 & 1 & 1 & 2 & 1 & 1 \\
\hline INN51_748S_CF_DL_India $\dagger$ & Cassia fistula/Delhi & 2006 & 1 & 2 & 2 & 1 & 1 \\
\hline INN52_748d_1_CF_DL_India & Cassia fistula/Delhi & 2006 & 1 & 5 & 2 & 1 & 1 \\
\hline INN53_748d_2__CF_DL_India $\dagger$ & Cassia fistula/Delhi & 2006 & 3 & 1 & 3 & 1 & 1 \\
\hline INN54_749S_1_CF_DL_India $\dagger$ & Cassia fistula/Delhi & 2006 & 1 & 1 & 2 & 1 & 1 \\
\hline INN55_749S_2_CF_DL_India & Cassia fistula/Delhi & 2006 & 1 & 1 & 2 & 1 & 1 \\
\hline INN56_750S_1_CF_DL_India & Cassia fistula/Delhi & 2006 & 1 & 7 & 2 & 1 & 1 \\
\hline INN57_750S_2_CF_DL_India & Cassia fistula/Delhi & 2006 & 1 & 1 & 2 & 1 & 1 \\
\hline INN58_751d_CF_DL_India & Cassia fistula/Delhi & 2006 & 1 & 1 & 2 & 1 & 1 \\
\hline INN59_753d_1_CF_DL_India & Cassia fistula/Delhi & 2006 & 1 & 1 & 2 & 1 & 1 \\
\hline INN60_753d_2_CF_DL_India $\dagger$ & Cassia fistula/Delhi & 2006 & 1 & 1 & 2 & 1 & 1 \\
\hline INN61_757d_CF_DL_India & Cassia fistula/Delhi & 2006 & 2 & 3 & 2 & 1 & 1 \\
\hline INN62_272DDD'_FR_DL_India $\dagger$ & Ficus religiosa/Delhi & 2003 & 1 & 1 & 4 & 1 & 1 \\
\hline INN63_790S_1_A_SC_UP_India & Syzygium cumini/Bulandshahar & 2006 & 1 & 1 & 2 & 1 & 1 \\
\hline INN64_806S_1_AI_UP_India $\dagger$ & Azadirachta indica/Bulandshahar & 2006 & 1 & 1 & 5 & 1 & 1 \\
\hline INN65_809S_1_AI_UP_India $\dagger$ & Azadirachta indica/Bulandshahar & 2006 & 2 & 1 & 2 & 1 & 1 \\
\hline INN66_713S_3_AI_UP_India & Azadirachta indica/Hathras & 2006 & 1 & 1 & 1 & 1 & 1 \\
\hline INN67_717S_AI_UP_India & Azadirachta indica/Hathras & 2006 & 1 & 1 & 2 & 1 & 1 \\
\hline INN68_425Sd-BB_SC_PB_India & Syzygium cumini/Amritsar & 2004 & 1 & 1 & 1 & 1 & 1 \\
\hline INN69_425SS-C_SC_PB_India & Syzygium cumini/Amritsar & 2004 & 1 & 1 & 2 & 1 & 1 \\
\hline INN70_425SS-BB_SC_PB_India & Syzygium cumini/Amritsar & 2004 & 2 & 1 & 2 & 1 & 1 \\
\hline INN71_428B-WC_SC_PB_India & Syzygium cumini/Amritsar & 2004 & 2 & 1 & 2 & 1 & 1 \\
\hline INN72_428B-WC-BB_SC_PB_India & Syzygium cumini/Amritsar & 2004 & 1 & 1 & 2 & 1 & 1 \\
\hline
\end{tabular}


Table 1. cont.

\begin{tabular}{|c|c|c|c|c|c|c|c|}
\hline $\begin{array}{l}\text { Strain ID } \\
\text { INN73_428B-WC-BBT_SC_PB_India }\end{array}$ & $\begin{array}{l}\text { Source tree/geographical origin } \\
\text { Syzygium cumini/Amritsar }\end{array}$ & $\frac{\text { Year isolated }}{2004}$ & \multicolumn{5}{|c|}{ Multilocus genotype ${ }^{\star}$} \\
\hline INN75_428B-WS-BB_SC_PB_India & Syzygium cumini/Amritsar & 2004 & 1 & 1 & 2 & 1 & 1 \\
\hline INN77_430SS-BB_SC_PB_India & Syzygium cumini/Amritsar & 2004 & 1 & 1 & 2 & 1 & 1 \\
\hline INN78_432WS-C_SC_PB_India & Syzygium cumini/Amritsar & 2004 & 1 & 3 & 2 & 1 & 1 \\
\hline INN79_433Sd-C_SC_PB_India & Syzygium cumini/Amritsar & 2004 & 1 & 1 & 2 & 1 & 1 \\
\hline INN82_439WS-C_SC_PB_India & Syzygium cumini/Amritsar & 2004 & 2 & 3 & 2 & 1 & 1 \\
\hline INN83_440SS-BB_SC_PB_India & Syzygium cumini/Amritsar & 2004 & 2 & 3 & 2 & 1 & 1 \\
\hline INN84_441Sd-BB_1_SC_PB_India & Syzygium cumini/Amritsar & 2004 & 1 & 1 & 2 & 1 & 1 \\
\hline INN85_441Sd-BB_2_SC_PB_India & Syzygium cumini/Amritsar & 2004 & 1 & 1 & 2 & 1 & 1 \\
\hline INN86_441S-BB_SC_PB_India & Syzygium cumini/Amritsar & 2004 & 1 & 1 & 2 & 1 & 1 \\
\hline INN95_782d_2_MI_HR_India & Mangifera indica/Amrouli & 2006 & 1 & 5 & 2 & 1 & 1 \\
\hline INN96_782d_3_MI_HR_India & Mangifera indica/Amrouli & 2006 & 3 & 1 & 3 & 1 & 1 \\
\hline INN97_782S_1_MI_HR_India & Mangifera indica/Amrouli & 2006 & 1 & 5 & 2 & 1 & 1 \\
\hline INN98_782S_2_MI_HR_India & Mangifera indica/Amrouli & 2006 & 1 & 1 & 2 & 1 & 1 \\
\hline
\end{tabular}

*Alleles for markers (from left to right) CAP1, FTR1, LAC, ITS, MtLrRNA.

$\dagger$ Indicates strains that have been tested for mating ability.

origins. Statistical significance for this test was derived by comparing the observed dataset to 10000 randomized datasets. During randomization, any linkage disequilibria present in the observed data were maintained. The statistic $\theta$ ranges from 0 (for no population differentiation due to frequent gene flow) to 1 (total isolation of the populations from each other). In the third analysis, we examined the relationship between genetic distance and geographical distance between populations, using the Isolation by Distance Web Service (http://ibdws.sdsu.edu/) (Jensen et al., 2005). A spatially limited gene flow would result in decreased genetic similarity between populations as their geographical distances increase, and this is commonly referred to as isolation by distance (IBD) (Wright, 1938). This test plots and correlates genetic distances and geographical distances between pairs of populations. The genetic distance measure used here is the standard Nei's genetic distance $(D)$ between populations (Nei, 1972), established based on either host tree species or geographical origin.

Mating assays. The abilities to mate and reproduce sexually were examined for representative strains in this study. Briefly, 16 strains representing all 15 multilocus genotypes (MGs) identified in this study were chosen (see below; these strains are marked in Table 1). Each of the 16 strains was mated with three standard mating testers for $C$. neoformans independently. The three testers were JEC20 (MATa), JEC21 (MAT $\alpha$ ) and H99 (MAT $\alpha)$. JEC20 and JEC21 belong to C. neoformans var. neoformans (serotype D) while H99 belongs to C. neoformans var. grubii (serotype A). To prepare for mating, strains were first grown on YEPD (yeast extract-peptone-dextrose) medium at room temperature for 3 days. Approximately equal numbers of cells from mating partners were then mixed on V8-Juice agar medium [5\% (v/v) V8 juice, $3 \mathrm{mM} \mathrm{KH_{2 }} \mathrm{PO}_{4}$ and $4 \%(\mathrm{w} / \mathrm{v})$ agar at $\mathrm{pH}$ 7]. The plates were then incubated in the absence of light at room temperature $\left(\sim 22{ }^{\circ} \mathrm{C}\right)$ for 21 days. For negative controls, we separately inoculated the three tester strains as well as the 16 representative strains from India onto V8-Juice agar medium and incubated under the same condition as described above for mating mixtures. For positive controls, we mated JEC20 and H99. In addition, we constructed several pairs of same-sex matings between strains from India (all MAT $\alpha$ ). Filamentation and basidiospore formation were recorded by light microscopy for each of the matings and controls.

\section{RESULTS}

\section{Molecular variation}

We were able to amplify all five DNA fragments for all the 78 isolates collected for this study. The results of PCR using the STE12 and STE20 gene primers showed that all isolates belonged to the $\alpha$ mating type. Our direct sequencing of PCR products identified no ambiguous nucleotide sites at any of the five genes for any of the 78 strains, consistent with haploidy. In total, we obtained 2912 nucleotides for each strain. The entire alignment had 11 parsimonyinformative sites and 5 parsimony-uninformative sites. No polymorphic site was observed within the sequenced regions for the ITS and mtLrRNA gene fragments. The GenBank accession numbers for the 390 DNA sequences are: CAP1, EU312204-EU312281; FTR1, EU312282EU312359; ITS, EU312360-EU312437; LAC, EU312438EU312515; $m t L r R N A$, EU312516-EU312593.

We used both maximum-parsimony and maximumlikelihood analyses to construct the evolutionary relationships among strains for each of the three gene loci that 
contained nucleotide polymorphisms, both individually (see supplementary Fig. S1, available with the online version of this paper) and in combination (Fig. 1). Both methods of analysis generated identical phylogenetic patterns. All the isolates clustered more closely with the C. neoformans var. grubii model strain H99 (serotype A) than with the $C$. neoformans var. neoformans model strain JEC21 (serotype D), consistent with our serological identification that all the strains analysed here belonged to serotype A. Aside from the LAC phylogeny, midpoint rooting was used to display the topologies of all the phylogenies to increase resolution among genotypes (Fig. S1 and Fig. 1). However, the topologies did not change when we rooted the trees using the orthologues from the serotype D strain JEC21 for either the CAP1 or the FTR1 gene tree. In each of the three polymorphic genetic loci, we saw no obvious evidence of strain clustering based on either geographical origin or host tree species (Fig. S1). Indeed, all major multilocus genotypes are shared among strains from multiple geographical regions or host tree species.

Among the 78 strains, the number of haplotypes for the individual gene fragments was as follows: four for $L A C$, three for CAP1, eight for FTR1 (Fig. S1), and one each for ITS and mtLrRNA (Table 1). In total, the entire dataset yielded 16 multilocus genotypes among the 78 strains (Fig. 1). Almost all the genotypes identified here were represented in the largest subpopulation, from Delhi (Fig. 1). The second-largest subpopulation (Amritsar) had two haplotypes for each of CAP1, FTR1 and LAC.

To compare the multilocus genotypes identified here with those reported previously, we compared our sequences with those in the GenBank using BLASTN. Our analyses identified that all our sequences had the closest matches to sequences of strains of $C$. neoformans var. grubii, consistent with our expectation. Of the three gene fragments analysed here, $L A C$ has been the most widely used in previous studies. As a result, a significant number of allelic sequences exist for the LAC gene in GenBank and some of these sequences have been used to define several molecular types within C. neoformans (Litvintseva et al., 2003 , 2006). We therefore analysed our sequences with eight representative sequences from the three different molecular types to determine the likely molecular type(s) of our strains. The eight representative sequences of the $L A C$ gene included five of VNI, two of VNB and one of VNII. Our analyses identified that all 78 strains showed the highest similarity to strains of the VNI molecular type (Fig. S1a).

\section{Lack of genetic differentiation between populations}

The $\theta$ value, a measure of population subdivision, showed no significant differentiation among geographical or hosttree-based populations and the observed value lies well within the distribution of the randomized datasets (only results based on geographical origins are shown in Fig. 2). The amount of genetic differentiation observed between populations based on either geographical location (Table 2 ) or host tree species (Table 3) was also measured by Nei's $D$ and we observed no significant difference between these populations. The lack of significant genetic differentiation is also supported by a lack of monophyletic pattern in the T-PTP tests based on either geographical origins or host tree species, with T-PTP values of 1.00 and 0.60 respectively. While we observed a positive correlation between geographical distance and pairwise population genetic distance, the correlation was statistically not significant (data not shown).

\section{Evidence for both clonality and recombination}

As described above, the evidence for clonality and recombination was examined using three tests. The first was the partition homogeneity test or incongruence length difference test. This test showed no statistically significant heterogeneity among the five markers used in this study. This test was conducted for the total sample of 78 strains as well as separately for each of the two largest geographical populations (Delhi and Amritsar). None of the samples showed any significant incongruence between genealogies from different markers [entire dataset $(n=78), P=0.27$; Delhi $(n=47), P=0.23$; Amritsar $(n=21), P=0.75]$. This result suggests that the overall population structure is consistent with significant clonality and clonal expansion. We also found other evidence for clonality based on the distribution of different genotypes between different geographical locations (Fig. 1). For example, two multilocus genotypes (MGs), MGIX and MGXI, were overrepresented in the total sample with 41 and 10 isolates each, both from different host trees and geographical areas (Fig. 1). Furthermore, with the exception of MGIV and MGXVI (Fig. 1), all the other genotypes in our study were represented in the strains from Delhi. The MGXVI genotype was recovered from three different cities: Amritsar, Bulandshahar and Hathras. The lone genotype MGIV was from Bulandshahar. While genotypes MGI, MGII, MGIII, MGVI, MGVIII, MGX, MGIV and MGXV were exclusive to Delhi, the remaining genotypes were each sampled from more than one geographical location. This pattern of distribution and the sharing of genotypes between different geographical locations are consistent with long-distance dispersal and clonal expansion within and among the geographical populations of C. neoformans var. grubii from India.

The second test assessed the overall associations among alleles at different loci. Our population genetic analyses revealed that both $I_{A}$ and RbarD values were low both for the total population sample as well as for the two largest subpopulations (Delhi and Amritsar) (Table 4). In fact, the null hypothesis of random recombination could not be rejected and the $I_{\mathrm{A}}$ values were well within the randomized recombining datasets (Fig. 3). We repeated these tests for 


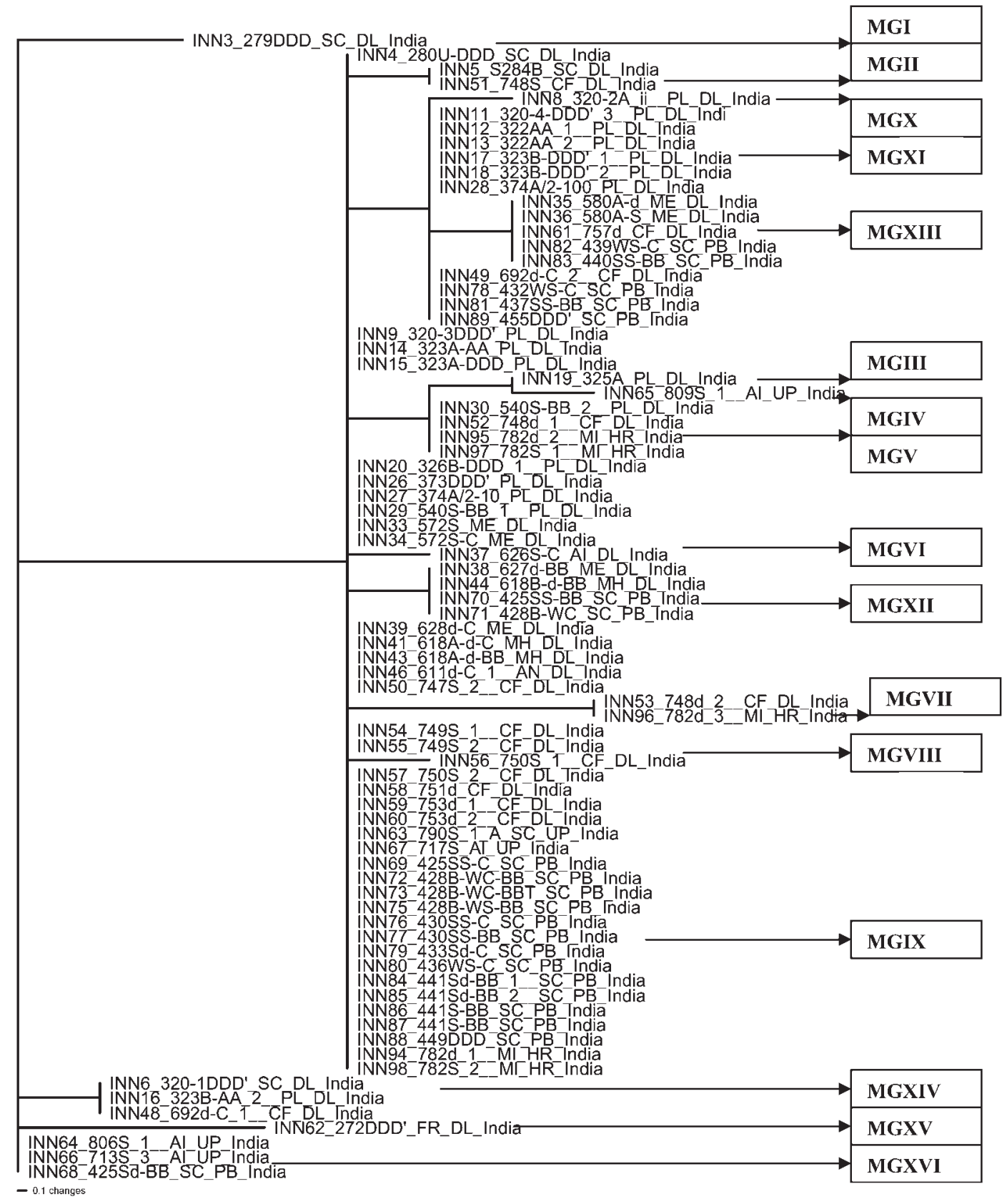

Fig. 1. Maximum-parsimony trees showing the relationships among 78 isolates of Cryptococcus neoformans var. grubii for the combined dataset using mid-point rooting (constructed using five markers: CAP1, FTR1, LAC, ITS and mtLrRNA). MPTs, most parsimonious trees; Cl, consistency index; Rl, retention index; HI, homoplasy index. Combined dataset (2912 bp aligned; 84 MPTs; tree length $=20 ; \mathrm{Cl}=0.850, \mathrm{RI}=0.953, \mathrm{HI}=0.150)$. The combined dataset showed a phylogenetic pattern that did not distinguish populations based on either host trees or geographical locations. Genotype labels MGI to MGXVI represent the different multilocus haploid genotypes found in this study based on the five gene fragments. The midpoint-rooted tree is identical in topology to that based on an outgroup-rooted tree using sequences from the serotype D strain JEC21. The midpoint-rooted tree is shown to increase the proportional lengths of branches for better visualization.

samples specific to each host tree species and all failed to reject the null hypothesis of random recombination. More importantly, our third test, using phylogenetic incompatibility, also found clear evidence for recombination.
Specifically, among three polymorphic loci, LAC, CAP1 and FTR1, we found all possible allelic combinations between pairs of loci for several alleles (raw data in Table 1 and examples shown in Table 5). Our results thus suggest 


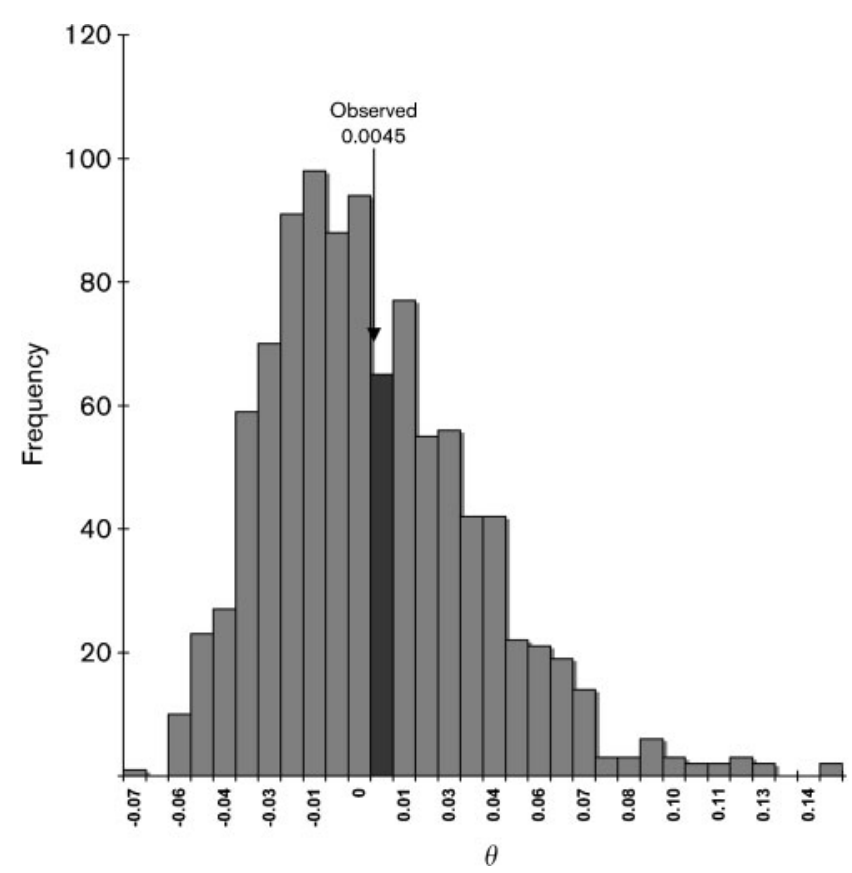

Fig. 2. Distribution of $\theta$ (a measure of genetic differentiation) among geographical populations of $C$. neoformans var. grubii from India. The $\theta$ value was well within the distribution of the permuted datasets that assumed no genetic differentiation $(P=0.366)$.

that recombination is occurring within all populations analysed in this study.

\section{Fertility of C. neoformans var. grubii strains from India}

Among the 48 crosses that we set up between 16 representative strains and the three testers, only seven successfully produced hyphal filaments and basidiospores. All seven were with strain JEC20 (the MATa mating tester) and none with JEC21 or H99. The results are consistent with our molecular determination of mating types and suggest that at least some of the strains are sexually fertile. While the positive control cross JEC20 $\times$ H99 mated successfully, none of the negative controls worked. In addition, after more than 3 weeks of incubation, none of the crosses between strains of the same sex, MAT , produced any hyphal filaments.

\section{DISCUSSION}

C. neoformans var. grubii is among the most important fungal pathogens in humans. Understanding its population structure and ecology in natural environments could have significant medical implications. In this study, our population-genetic analyses showed evidence for extensive gene flow among the environmental populations of this organism in India. Furthermore, we identified evidence for both clonality and recombination in this sample, which contained exclusively the MAT $\alpha$ mating type. Our results are consistent with the hypothesis that $C$. neoformans might be able to complete its sexual life cycle on decaying wood of several tree species in India. A recent study by Xue et al. (2007) demonstrated that mating and sexual reproduction between strains of opposite mating types can occur for both $C$. neoformans and $C$. gattii on the model plant Arabidopsis thaliana and a natural tree host of C. gattii, the eucalyptus.

All of the host tree species investigated in this study are native to India. These tree species included Syzygium cumini (Java plum/jamun tree), Polyalthia longifolia (Indian mast tree), Mimusops elengi (bullet wood), Azadirachta indica (neem tree), Manilkara hexandra (margosa), Acacia nilotica (thorn mimosa), Cassia fistula (golden shower tree) and Mangifera indica (mango). In particular, one tree species, $P$. longifolia, is found around many homes throughout India. All the trees from which $C$. neoformans var. grubii was sampled were very close to human habitation. In addition to these tree species, $C$. neoformans var. grubii has also been recovered from the hollows of other tree species such as Cassia grandis (pink shower tree), Senna multijuga (November shower tree) and Ficus microcarpa (fig tree) (Lazera et al., 1996). Our results demonstrated that the populations of $C$. neoformans var. grubii in different tree species are not genetically different and that there is frequent gene flow among different tree species. The spread of this micro-organism between different hosts could have been mediated by wind-aided dispersal of basidiospores or desiccated vegetative cells. The five cities from where the strains were isolated in this study

Table 2. Genetic differences among populations of C. neoformans var. grubii in India: Nei's genetic distance $D$ between geographical populations

\begin{tabular}{|lccccc|}
\hline Geographical region & $\begin{array}{c}\text { Delhi } \\
(\boldsymbol{n}=\mathbf{4 7})\end{array}$ & $\begin{array}{c}\text { Bulandshahar } \\
(\boldsymbol{n}=\mathbf{3})\end{array}$ & $\begin{array}{c}\text { Hathras } \\
(\boldsymbol{n}=\mathbf{2})\end{array}$ & $\begin{array}{c}\text { Amritsar } \\
(\boldsymbol{n}=\mathbf{2 1})\end{array}$ & $\begin{array}{c}\text { Amrouli } \\
(\boldsymbol{n}=\mathbf{5})\end{array}$ \\
\hline Delhi $28^{\circ} 36^{\prime} 0^{\prime \prime} \mathrm{E} 77^{\circ} 19^{\prime} 0^{\prime \prime} \mathrm{N}$ & 0 & & & & \\
Bulandshahar $28^{\circ} 24^{\prime} 0^{\prime \prime} \mathrm{E} 77^{\circ} 51^{\prime} 0^{\prime \prime} \mathrm{N}$ & 0.053 & 0 & & & \\
Hathras $27^{\circ} 36^{\prime} 0^{\prime \prime} \mathrm{E} 78^{\circ} 3^{\prime} 0^{\prime \prime} \mathrm{N}$ & 0.076 & 0.058 & 0 & & \\
Amritsar $31^{\circ} 37^{\prime} 59^{\prime \prime} \mathrm{E} 74^{\circ} 51^{\prime} 56^{\prime \prime} \mathrm{N}$ & 0.007 & 0.047 & 0.070 & 0 & \\
Amrouli $28^{\circ} 25^{\prime} 59^{\prime \prime} \mathrm{E} 77^{\circ} 19^{\prime} 0^{\prime \prime} \mathrm{N}$ & 0.038 & 0.084 & 0.095 & 0.048 & 0 \\
\hline
\end{tabular}


Table 3. Genetic differences among populations of C. neoformans var. grubii in India: Nei's genetic distance $D$ between populations from different host tree species

\begin{tabular}{|lcccccc|}
\hline Host tree species & $\begin{array}{c}\text { Syzygium } \\
\text { cumini } \\
(\boldsymbol{n}=\mathbf{2 6})\end{array}$ & $\begin{array}{c}\text { Polyalthia } \\
\text { longfolia } \\
(\boldsymbol{n}=\mathbf{1 4})\end{array}$ & $\begin{array}{c}\text { Mimusops } \\
\text { elengi } \\
(\boldsymbol{n}=\mathbf{4})\end{array}$ & $\begin{array}{c}\text { Azadirachta } \\
\text { indica } \\
(\boldsymbol{n}=7)\end{array}$ & $\begin{array}{c}\text { Manilkara } \\
\text { hexandra } \\
(\boldsymbol{n}=\mathbf{3})\end{array}$ & $\begin{array}{c}\text { Cassia } \\
\text { fistula } \\
(\boldsymbol{n}=\mathbf{1 4})\end{array}$ \\
$\begin{array}{l}\text { Syzygium cumini } \\
\text { Polyalthia longfolia }\end{array}$ & 0 & & & & \\
Mimusops elengi & 0.022 & 0 & & & \\
Azadirachta indica & 0.050 & 0.054 & 0 & & & \\
Manilkara hexandra & 0.024 & 0.068 & 0.079 & 0 & & \\
Cassia fistula & 0.024 & 0.082 & 0.065 & 0.031 & 0 & 0.044 \\
Mangifera indica & 0.007 & 0.013 & 0.059 & 0.035 & 0.067 & 0.028 \\
\hline
\end{tabular}

are among the most densely populated regions in India. This is especially true of Delhi, where human population density and migration are both very high. The high rate of human migration could also contribute to the dispersal of C. neoformans var. grubii among different locations. In addition, tree-dwelling animals such as birds and squirrels can potentially aid in the spread and dispersal of this organism. At the global level, there is evidence for limited genetic differentiation and frequent gene flow among geographical populations of C. neoformans var. grubii (Xu et al., 2000; Litvintseva et al., 2003, 2006). Short- and longdistance dispersals have been reported previously for many fungal species, including saprobes, and plant and animal pathogens (Xu, 2005b).

The limited genetic variation found here among strains from the different hosts and geographical regions suggests that the Indian population of C. neoformans var. grubii might be of recent origin and/or that the dispersals were very frequent and recent. While the widespread overrepresentation of certain genotypes is consistent with clonality, the identification of phylogenetic incompatibility and low $I_{\mathrm{A}}$ values also suggested the presence of recombination in the Indian environmental population of C. neoformans var. grubii. These results are consistent with the structures of many microbial populations, characterized by unambiguous recombination, clonal dispersal and expansion of a few genotypes (e.g. Maynard-Smith et al., 1993; Xu, 2005b).

The evidence for recombination in a MAT $\alpha$ population of C. neoformans var. grubii is surprising. Previous analyses of samples of $C$. neoformans var. grubii have indicated that clonality was the dominant feature of reproduction in natural populations. This was somewhat expected because in most previous studies, the strains analysed from either human or environmental sources belonged to mating type $\alpha$. There were two exceptions where evidence for recombination was detected in C. neoformans var. grubii. The first was an analysis of 14 hybrid strains of serotypes $\mathrm{AD}$ where both mating types $a$ and $\alpha$ strains from serotypes $\mathrm{A}$ and $\mathrm{D}$ were reconstructed using gene genealogies. The reconstructed serotype $\mathrm{A}$ and $\mathrm{D}$ populations both showed evidence of recombination (Xu \& Mitchell, 2003). The second example was from humans in Botswana, where both mating types were found and, not surprisingly, evidence for recombination as well (Litvintseva et al., 2003).

Our current study is unique in that there was clear evidence for recombination in a sample where all the isolates belonged to one mating type, MATa. At present, the detailed mechanism for our observed recombination is not known. However, there are several possibilities. The first is that, unlike in most previous studies (e.g. Litvintseva et al., 2003; Xu et al., 2000), the samples analysed here were all from a geographically more restricted region, in northwestern India. The close geographical proximity could have contributed to frequent mating and genetic mixing, resulting in recombinant genotypes. The second is that all strains analysed here are genetically very similar to each other. In fact, all 78 strains here were found to belong to the same molecular type, VNI. Strains with similar

Table 4. Multilocus linkage disequilibrium analyses for samples of C. neoformans var. grubii from India (subpopulations with sample sizes greater than 5 are also shown)

\begin{tabular}{|lccc|}
\hline Population & $\boldsymbol{I}_{\mathbf{A}}^{*}(\boldsymbol{P}$ value $)$ & RbarD $(\boldsymbol{P}$ value $)$ & Phylogenetic incompatibility $(\boldsymbol{P}$ value $)$ \\
\hline Total $(n=78)$ & $0.1433(0.138)$ & $0.0360(0.138)$ & $0.5(<0.0001)$ \\
Delhi $(n=47)$ & $0.1519(0.102)$ & $0.0385(0.102)$ & $0.7(<0.0001)$ \\
Amritsar $(n=21)$ & $0.0454(0.363)$ & $0.0114(0.363)$ & $0.8(0.108)$ \\
\hline
\end{tabular}

${ }^{\star} \mathrm{I}_{\mathrm{A}}$, index of association. 


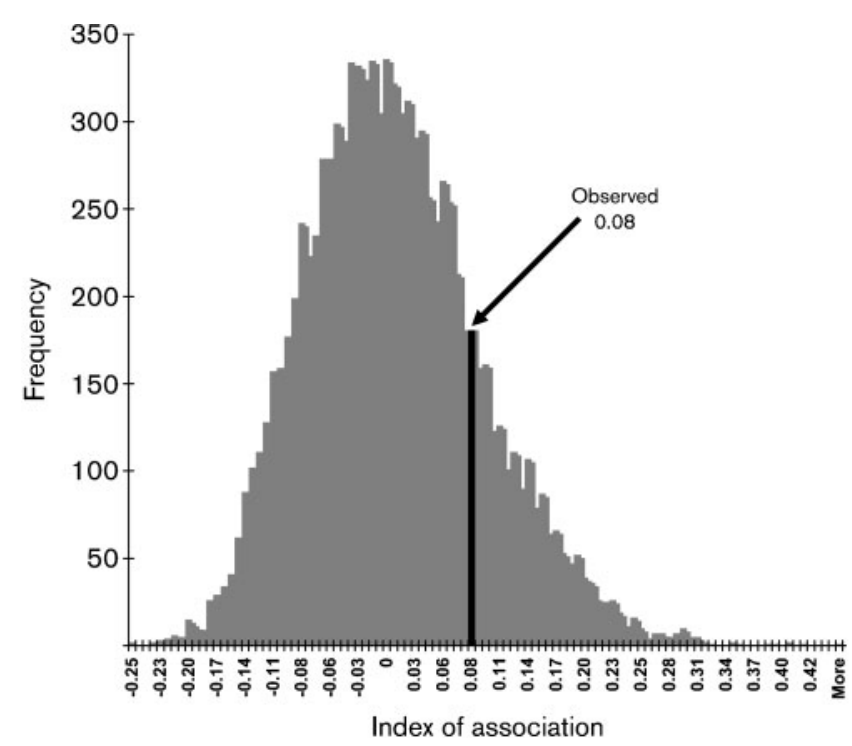

Fig. 3. Distribution of $I_{\mathrm{A}}$ values. The observed index of association $\left(I_{A}\right)$ value was within the distribution of $I_{A}$ obtained from 10000 artificially recombined datasets $(P=0.1816)$.

genotypes might have a greater opportunity of producing viable genetic recombinants capable of surviving in nature, as suggested previously by Campbell et al. (2005). The third is that the environmental niche, tree hollows, might be highly conducive to mating. A recent study indicated that both $C$. neoformans and C. gattii could complete their sexual reproductive life cycle in plants (Xue et al., 2007). Most previous studies of environmental samples of $C$. neoformans var. grubii have focused on pigeon guano. Whether populations of $C$. neoformans var. grubii from different ecological niches (e.g. pigeon guano and soil) in

Table 5. Allelic combinations showing evidence of recombination in the environmental population of C. neoformans var. grubii from India

(a) All four possible allelic combinations are found between alleles 1 and 3 of locus FTR1 and alleles 1 and 2 of locus LAC. (b) All six possible allelic combinations are found between alleles 1,3 , and 5 of locus FTR 1 and alleles 1 and 2 of locus CAP1. The number of strains for each genotype is shown in the table.

\begin{tabular}{|lcc|}
\hline (a) & LAC (allele 1) & LAC (allele 2) \\
\hline FTR1 (allele 1) & 4 & 42 \\
FTR1 (allele 3) & 3 & 14 \\
\hline (b) & CAP1 (allele 1) & CAP1 (allele 2) \\
\hline FTR1 (allele 1) & 43 & 4 \\
FTR1 (allele 3) & 11 & 5 \\
FTR1 (allele 5) & 4 & 1 \\
\hline
\end{tabular}

India show population structures similar to that from tree hollows remains to be examined.

The sexual recombination detected here could be achieved through one of two pathways. The first possibility is that recombination was due to same-sex mating between MAT $\alpha$ strains in natural populations of $C$. neoformans var. grubii. Same-sex mating has been observed in the laboratory (Lin et al., 2005; Yan et al., 2007) and in nature between strains of serotypes A and D (Lin et al., 2007). The second hypothesis is that the recombination observed here was between strains of opposite mating types. Even though we were unable to isolate any MATa strains from India, it is possible that MATa strains are there but are very rare in nature in India and difficult to isolate. Our result that some of these isolates can mate with MATa but not MAT $\alpha$ strains in the laboratory suggests that these MATa strains might be present in the environment in India to help maintain the sexual fertility of MAT $\alpha$ strains. However, more extensive sampling would be required to distinguish between these two hypotheses. We would like to emphasize that the evidence for recombination was found not only for the whole sample but also for the subpopulations in Delhi and Amritsar. This finding suggests that many geographical regions in India (and potentially other places in other parts of the world) could be hotspots for sexual mating and the dispersal of basidiospores of this organism.

A combined sexual and asexual mode of reproduction is common for many microbial species, including many human pathogens (Xu, 2005b). It was recently shown that sexual reproduction could incur a cost in fitness $(\mathrm{Xu}, 2005 \mathrm{a})$. It is also well known that sexual reproduction can produce a large number of recombinant genotypes that could speed up adaptation to novel environments (Weismann, 1904; Goddard et al., 2005). In C. neoformans, while mating could occur between strains of the same or different sexes, it remains to be seen if the two alternative forms of mating have different adaptive significance. In addition, we know very little of the specific conditions in nature under which this organism undergoes same-sex mating as opposed to mating between opposite mating types. In the laboratory setting, low nitrogen, low moisture and ambient temperature are conducive to mating. The nutritional and other physicochemical features of tree hollows from where C. neoformans were isolated are unknown at present. Ecological and physiological studies of natural environments conducive for C. neoformans mating will provide further insights into the mechanisms of evolution and population structuring of this important fungal pathogen.

\section{ACKNOWLEDGEMENTS}

This work was supported by grants from the Natural Science and Engineering Research Council of Canada (J.X.), Genome Canada (J.X.), and the Premier's Research Excellence Award (J.X.). We thank the Indian National Science Academy for the award of a Hony Scientist position to H.S. R. and to the Department of Science \& Technology, New Delhi, for the award of a Young Scientist research grant to A. C. 


\section{REFERENCES}

Agapow, P.-M. \& Burt, A. (2001). Indices of multilocus linkage disequilibrium. Mol Ecol Notes 1, 101-102.

Avise, J. C. (1994). Molecular Markers, Natural History and Evolution. New York: Chapman \& Hall.

Barker, F. K. \& Lutzoni, F. M. (2002). The utility of the incongruence length difference test. Syst Biol 51, 625-637.

Bennett, J. E., Kwon-Chung, K. J. \& Howard, D. H. (1977). Epidemiologic differences among serotypes of Cryptococcus neoformans. Am J Epidemiol 105, 582-586.

Campbell, L. T., Currie, B. J., Krockenberger, M., Malik, R., Meyer, W., Heitman, J. \& Carter, D. (2005). Clonality and recombination in genetically differentiated subgroups of Cryptococcus gattii. Eukaryot Cell 4, 1403-1409.

Casadevall, A. \& Perfect, J. R. (1998). Cryptococcus neoformans. Washington, DC: ASM Press.

Casadevall, A., Steenbergen, J. N. \& Nosanchuk, J. D. (2003). Readymade virulence and dual use virulence factors in pathogenic environmental fungi - the Cryptococcus neoformans paradigm. Curr Opin Microbiol 6, 332-337.

Faith, D. P. (1991). Cladistic permutation tests for monophyly and nonmonophyly. Syst Zool 40, 366-375.

Farris, J. S., Källersjö, M., Kluge, A. G. \& Bult, C. (1994). Testing significance of incongruence. Cladistics 10, 315-319.

Fraser, J. A., Giles, S. S., Wenink, E. C., Geunes-Boyer, S. G., Wright, J. R., Diezmann, S., Allen, A., Stajich, J. E., Dietrich, F. S. \& other authors (2005). Same-sex mating and the origin of the Vancouver Island Cryptococcus gattii outbreak. Nature 437, 1360-1364.

Galtier, N., Gouy, M. \& Gautier, C. (1996). SeaView and PHYLO_WIN, two graphic tools for sequence alignment and molecular phylogeny. Comput Appl Biosci 12, 543-548.

Goddard, M. R., Godfray, H. C. J. \& Burt, A. (2005). Sex increases the efficacy of natural selection in experimental yeast populations. Nature 434, 636-640.

Jensen, J. L., Bohonak, A. J. \& Kelley, S. T. (2005). Isolation by distance, web service v.3.14. BMC Genet 6, 13.

Keller, S. M., Viviani, M. A., Esposto, M. C., Cogliati, M. \& Wickes, B. L. (2003). Molecular and genetic characterization of a serotype A MATa Cryptococcus neoformans isolate. Microbiology 149, 131-142.

Kidd, S. E., Guo, H., Bartlett, K. H., Xu, J. \& Kronstad, J. W. (2005). Comparative gene genealogies indicate that two clonal lineages of Cryptococcus gattii in British Columbia resemble strains from other geographical areas. Eukaryot Cell 4, 1629-1638.

Kwon-Chung, K. J. (1975). A new genus, Filobasidiella, the perfect state of Cryptococcus neoformans. Mycologia 67, 1197-1200.

Kwon-Chung, K. J. (1976). Morphogenesis of Filobasidiella neoformans, the sexual state of Cryptococcus neoformans. Mycologia 68, 821-833.

Kwon-Chung, K. J. \& Bennett, J. E. (1978). Distribution of $\alpha$ and $a$ mating types of Cryptococcus neoformans among natural and clinical isolates. Am J Epidemiol 108, 337-340.

Kwon-Chung, K. J., Wickes, B. L., Stockman, L., Roberts, G. D., Ellis, D. \& Howard, D. H. (1992). Virulence, serotype, and molecular characteristics of environmental strains of Cryptococcus neoformans var. gattii. Infect Immun 60, 1869-1874.

Lazera, M. S., Pires, F. D., Camillo-Coura, L., Nishikawa, M. M., Bezerra, C. C., Trilles, L. \& Wanke, B. (1996). Natural habitat of Cryptococcus neoformans var. neoformans in decaying wood forming hollows in living trees. J Med Vet Mycol 34, 127-131.
Lin, X., Hull, C. M. \& Heitman, J. (2005). Sexual reproduction between partners of the same mating type in Cryptococcus neoformans. Nature 434, 1017-1021.

Lin, X., Huang, J. C., Mitchell, T. G. \& Heitman, J. (2006). Virulence attributes and hyphal growth of $C$. neoformans are quantitative traits and the MATa allele enhances filamentation. PLoS Genet 2, e187.

Lin, X., Litvintseva, A. P., Nielsen, K., Patel, S., Floyd, A., Mitchell, T. G. \& Heitman, J. (2007). $\alpha \mathrm{AD} \alpha$ hybrids of Cryptococcus neoformans: evidence of same-sex mating in nature and hybrid fitness. PLoS Genet 3, 1975-1990.

Litvintseva, A. P., Marra, R. E., Nielsen, K., Heitman, J., Vilgalys, R. J. \& Mitchell, T. G. (2003). Evidence of sexual recombination among Cryptococcus neoformans serotype A isolates in sub-Saharan Africa. Eukaryot Cell 2, 1162-1168.

Litvintseva, A. P., Thakur, R., Vilgalys, R. \& Mitchell, T. G. (2006). Multilocus sequence typing reveals three genetic subpopulations of Cryptococcus neoformans var. grubii (serotype A), including a unique population in Botswana. Genetics 172, 2223-2238.

Loftus, B. J., Fung, E., Roncaglia, P., Rowley, D., Amedeo, P., Bruno, D., Vamathevan, J., Miranda, M., Anderson, I. J. \& other authors (2005). The genome of the basidiomycetous yeast and human pathogen Cryptococcus neoformans. Science 307, 1321-1324.

Maynard-Smith, J., Smith, N. H., O'Rourke, M. \& Spratt, B. G. (1993). How clonal are bacteria? Proc Natl Acad Sci U S A 90, 4384-4388.

Nei, M. (1972). Genetic distance between populations. Am Nat 106, 283-292.

Nielsen, K., Cox, G. M., Wang, P., Toffaletti, D. L., Perfect, J. R. \& Heitman, J. (2003). Sexual cycle of Cryptococcus neoformans variety grubii and virulence of congenic $a$ and $\alpha$ isolates. Infect Immun 71, 4831-4841.

Randhawa, H. S., Kowshik, T. \& Khan, Z. U. (2003). Decayed wood of Syzygium cumini and Ficus religiosa living trees in Delhi/New Delhi metropolitan area as natural habitat of Cryptococcus neoformans. Med Mycol 41, 199-209.

Randhawa, H. S., Kowshik, T. \& Khan, Z. U. (2005). Efficacy of swabbing versus a conventional technique for isolation of Cryptococcus neoformans from decayed wood in tree trunk hollows. Med Mycol 43, 67-71.

Reimão, J. Q., Drummond, E. D., Terceti, M. de. S., Lyon, J. P., Franco, M. C. \& de Siqueira, A. M. (2007). Isolation of Cryptococcus neoformans from hollows of living trees in the city of Alfenas, MG, Brazil. Mycoses 50, 261-264.

Swofford, D. L. (1996). Phylogenetic inference. In Molecular Systematics, pp. 407-514. Edited by D. Hill, C. Moritz \& B. Mable. Sunderland, MA: Sinauer Associates.

Thompson, J. D., Gibson, T. J., Plewniak, F., Jeanmougin, F. \& Higgins, D. G. (1997). The CLUSTAL_X-Windows interface: flexible strategies for multiple sequence alignment aided by quality analysis tools. Nucleic Acids Res 24, 4876-4882.

Tintelnot, K., Lemmer, K., Losert, H., Schar, G. \& Polak, A. (2004). Follow-up of epidemiological data of cryptococcosis in Austria, Germany and Switzerland with special focus on the characterization of clinical isolates. Mycoses 47, 455-464.

Weir, B. S. (1996). Genetic Data Analysis II. Sunderland: Sinauer.

Weismann, A. (1904). The Evolution Theory, 2 vols. Translated from the 1904 2nd German edition by J. A. Thomson \& M. R. Thomson. London: Edward Arnold.

Wright, S. (1938). Size of population and breeding structure in relation to evolution. Science 87, 430-431. 
Xu, J. (2005a). Cost of interacting with sexual partners in a facultative sexual microbe. Genetics 171, 1597-1604.

Xu, J. (2005b). Evolutionary Genetics of Fungi. UK: Horizon Bioscience.

Xu, J. \& Mitchell, T. G. (2003). Comparative gene genealogical analyses of strains of serotype $\mathrm{AD}$ identify recombination in populations of serotypes A and D in the human pathogenic yeast Cryptococcus neoformans. Microbiology 149, 2147-2154.

Xu, J., Mitchell, T. G. \& Vilgalys, R. (1999). PCR-restriction fragment length polymorphism (RFLP) analyses reveal both extensive clonality and local genetic differentiation in Candida albicans. Mol Ecol 8, 59-73.

Xu, J., Vilgalys, R. \& Mitchell, T. D. (2000). Multiple gene genealogies reveal recent dispersion and hybridization in the human pathogenic fungus Cryptococcus neoformans. Mol Ecol 9, 1471-1481.
Xue, C., Tada, Y., Dong, X. \& Heitman, J. (2007). The human fungal pathogen Cryptococcus can complete its sexual cycle during a pathogenic association with plants. Cell Host Microbe 1, 263-273.

Yan, Z., Li, X. \& Xu, J. (2002). Geographic distribution of mating type alleles of Cryptococcus neoformans in four areas of the United States. J Clin Microbiol 40, 965-972.

Yan, Z., Hull, C. M., Sun, S., Heitman, J. \& Xu, J. (2007). The mating type-specific homeodomain genes $S X I 1 \alpha$ and $S X I 2 a$ coordinately control uniparental mitochondrial inheritance in Cryptococcus neoformans. Curr Genet 51, 187-195.

Edited by: N. L. Glass 